\title{
Investigation of the Omaha System for Dentistry
}

M.W. Jurkovich1; M. Ophaug2; S. Salberg²; K. Monsen ${ }^{3}$

${ }^{1}$ St. Anthony, Minnesota, United States; ${ }^{2}$ University of Minnesota, Nursing Informatics, Minneapolis, Minnesota, United States; 3University of Minnesota School of Nursing

\section{Keywords}

Omaha system, dentistry, electronic health records, problem classification, SNOMED CT

\section{Summary}

Objective: Today, dentists and hygienists have inadequate tools to identify contributing factors to dental disease, diagnosis of disease or to document outcomes in a standardized and machine readable format. Increasing demand to find the most effective care methodologies make the development of further terminologies for dentistry more urgent. Preventive care is the focus of early efforts to define best practices. We reviewed one possibility with a history of public health documentation that might assist in these early efforts at identifying best practices.

Methods: This paper examines, through a survey of dentists, the Omaha System Problem Classification Scheme. The survey requested that dentists rate the usefulness of knowing about specific signs and symptoms for each of the 42 problems within the Problem list of the Omaha System. Results: Using a weighted scoring system, 22 of the 42 problems received over $50 \%$ of the possible maximum score and 30 of the 42 problems received at least $25 \%$ of the possible points. Conclusion: These findings suggests that further evaluation of The Omaha System, may be useful to dentistry. At a minimum, the survey provides additional information about non-physiological problems, signs, and symptoms that may be appropriate for documentation purposes within an electronic health record (EHR) used in dentistry.

\section{Correspondence to:}

Mark W Jurkovich DDS, MBA, MHI

3205 Skycroft Drive

St. Anthony, MN. 55418-2551

Email: jurko003@umn.edu

\author{
Appl Clin Inform 2014; 5: 491-502 \\ DOI: 10.4338/ACI-2014-01-RA-0001 \\ received: January 20, 2014 \\ accepted: April 10, 2014 \\ published: May 21, 2014 \\ Citation: Jurkovich MW, Ophaug M, Salberg S, \\ Monsen K. Investigation of the Omaha System for \\ Dentistry. Appl Clin Inf 2014; 5: 491-502 \\ http://dx.doi.org/10.4338/ACI-2014-01-RA-0001
}




\section{Introduction}

The need for a terminology for dentistry in electronic health records (EHRs) is evident $[1,2]$. There is a significant push from within and outside of the profession to improve documentation for both research and recordkeeping purposes [3].

Dental documentation differs from documentation in other areas of health care in some significant ways. There has been limited need for dentists to document a diagnosis for a high percentage of the care delivered. Diagnostic and preventive services account for an overwhelming majority of all services provided [3]. There is no efficient way to relate this care to disease reduction or to identify which procedures might produce the greatest improvement in oral health. Reports and studies indicate that there is a significant disparity in disease and utilization based on economic status and special needs $[4,5]$. Types of documentation related to these broader findings are not currently available in dental terminologies today but are within The Omaha System.

Additionally, dental professionals generally consider good home care critical in reducing dental disease. Dentistry has no machine readable methodology for representation of home care. The Omaha System includes a method to document such interventions and how well they may be working.

Knowledge representation in dentistry has primarily been limited to a procedure coding system, Current Dental Terminology (CDT13), for documentation within dentist's patient records [2, 6]. CDT is designed to allow for documentation and billing of services provided. It contains less than 700 codes, is near universal within the United States, and has been used for over 25 years. Though revisions, additions, and deletions are considered each year, changes are quite limited year to year, suggesting that it is meeting the needs of both payers for billing purposes and dentists for documentation of services provided.

Diagnosis codes and outcomes measurements are not part of CDT. ICD9 and the coming ICD10 do contain some dental diagnostic codes, but they are relatively limited in their usefulness, particularly in relation to preventive procedures. With no requirement to submit a diagnostic code, dentistry has not adopted this as either a standard or an expectation. With the exception of procedures, most information is in a narrative form only. This makes research into best practices and any outcomes based initiatives difficult and of limited value [7]. General dentists, in particular, seldom use other interface or reference terminologies.

Dentistry has a very limited ability to relate outcomes to services provided due to its lack of recorded diagnostic codes [2] and the general lack of standardized forms of documenting some of the factors identified above. Additionally, a widely held perception within the profession is that patients may not select the best available treatment option and/or obtain it at the optimum time, frequently due to financial limitations [9]. This would also impact outcomes research. The utilization of a system that identifies pertinent socioeconomic factors, behaviors and, potentially, other considerations may provide a method to overcome this problem.

Electronic health records systems used in dental practices have only recently been adapted to allow for inclusion of diagnosis codes. There is no standard method for relating this information to procedures performed. Much like other areas of health care, dentistry will need multiple representation schema to provide for comprehensive, machine readable documentation.

Besides ICD9, two coding systems, EZCodes and SNODENT (a subset of SNOMED CT) are available for documenting diagnosis but have limited ability to represent oral health status. Both EZCodes and SNODENT are in the process of being totally represented within SNOMED CT, which provides a significant basis of substances, findings, observations, and materials used in dentistry as well as diagnosis or disorders. However, no dental EHR currently used in the United States utilizes SNOMED CT comprehensively and there are no reasonable alternatives available at this time.

\section{The Omaha System and Dentistry}

The Omaha System is a methodology that allows for documentation of an entire care continuum. It has a Problem Classification Scheme, an Intervention Scheme and a Problem Rating Scale for Outcomes. The Problem Classification Scheme consists of four domains: Environmental, Psychosocial, 
Physiological, and Health-Related Behaviors. The Omaha System's inclusion of an Environmental and Psychosocial Domain within its classification scheme may allow for more differentiation that is considered to be contributory to dental health status $[4,5]$. These factors are not commonly represented within the medical history or demographics recorded and documented by dental clinics within their electronic record systems.

The Omaha System's developed relationships between problem classification, interventions, and outcomes is unique. It may be a tool for representing the large portion of dentistry that deals with preventive care, interventions, and home care through its inclusion of the problem rating scale for outcomes.

An example of the Omaha System Problem Classification Scheme with the problem oral health and its related signs/symptoms can be seen in Table 1. The Intervention Scheme has 4 categories, one of which would readily allow documentation using current CDT codes. The Problem Rating Scale for Outcomes is the final component of the Omaha System [10, 11].

An example of the Omaha System Problem Classification and Intervention Scheme for one of the most common issues, dental caries, is shown in $>$ Table 2. It is an indicator of the limited ability of the Omaha System to provide documentation for representing the granularity of dental disorders that may require interventions not associated with diagnostic and preventive therapies.

It is important to recognize that the Omaha System has been primarily used by nursing and public health. It has been applied effectively to inpatient and home care settings, most frequently by nurses $[10,11]$. Therefore, elements within dentistry may not be as easily captured using the Omaha System. The Problem Classification Scheme and Intervention Scheme list may need more specificity for acceptance within the dental community, if attempts are made to use it independently of other coding schema and broadly for all elements of recognized oral disease and therapies.

Dental caries and periodontal disease are identified as being very common conditions [5]. They are also thought to be highly preventable. Water fluoridation has specifically resulted in a significant reduction of dental caries, supporting the idea that these common diseases can be prevented. Thus, selection of a system used in public health, such as the Omaha System, may have merit in helping dentistry to research its public health initiatives to prevent disease, but may not allow it to be used comprehensively.

The Omaha System has been reviewed for reliability, validity and usefulness as a whole, which makes it an attractive option for possible application in other settings. Several non-dental management systems already include the Omaha System for knowledge representation, making it a reasonable alternative for software development $[10,11]$.

This research report is a first step to identifying whether a specific system used in other health care settings might work for research and development of diagnostic and preventive best practices in general dentistry. It summarizes the results of a survey of dentists regarding the Problem Classification Scheme of the Omaha System. Further study, involving the intervention scheme and problem rating scale for outcomes would be essential prior to any decision to implement the addition of this terminology to the dental setting.

\section{Methods}

The identification of various problems, signs, and symptoms that may influence the delivery of dental care and outcomes was determined through a survey of dentists using the Problem Classification Scheme of the Omaha System [10,11]. The use of the Intervention Scheme and Problem Rating Scale for Outcomes was not addressed.

A brief letter ( $>$ Appendix A) was developed to introduce the rationale to potential participating dentists which included a link to the survey site, SurveyMonkey, for ease of access [12]. This letter was sent via email to 183 dentists from various parts of the United States, nine of which were returned due to inaccurate email addresses. The link was open for 12 days, and the estimated time for completion was 15 to 20 minutes. No incentives were offered and all responses remained anonymous. Forty-two dentists completed the survey, resulting in a return rate of $24 \%$.

The survey itself was not random. Dentists belonging to a large multi-location group practice and others known to have obtained extensive continuing education were asked to respond. Additionally, 
no request was made of the dentists to state their specialty, so it is possible that a single group could have a heavier influence than their presence within the profession.

Participants were given three choices from which to select with regard to the sign or symptom for each of the 42 problems within the Omaha Problem Classification Scheme [10, 11]. Only one choice per sign/symptom was allowed. For each item listed, they were asked to consider

1. whether they would use the information,

2. whether others might use the information, or

3. whether they felt that the information was not useful in dental practice when providing care or determining a treatment plan.

Much discussion took place among the authors regarding the verbiage of the second option, "others might use". The authors recognized that a specific dentist might not use a certain sign or symptom, but allowed a dentist to select information that he/she thought other colleagues may find useful.

\section{Results}

A scoring system was applied to the raw data, assigning three points for each time a participant selected "I would use" and one point for each "others may use", as the authors felt that a heavier weight was justified for those signs and symptoms identified as most useful by the study participants. The Omaha System, as currently developed, requires the inclusion of all signs and symptoms within a specific problem. Thus, although some of the individual signs and symptoms within a problem may have rated quite low, their inclusion in a projected tool is necessary when considering use of the Omaha System comprehensively. Total scores were calculated for each sign and symptom and are listed in $>$ Table 3 . These totals were then divided by the number of signs and symptoms within each problem to allow for equally weighted scores for each problem. This allowed for comparison of each of the problems on an evenly weighted basis to identify those problems dentists rated more relevant.

The weighted averages seen in $>$ Table 3 display those problems that dentists considered most important in providing care or determining a treatment plan. The maximum weighted score any problem could receive is 126 , with three points for each "would use" multiplied by the number of people answering the survey up to 42 people. The minimum score was zero. The compilation indicates some natural breakpoints. With the exception of the break immediately following "Oral Health" which, as expected, scored extremely high, (123.86 as opposed to an absolute maximum of 126), the next significant division occurs near the midpoint of possible scores immediately following the Mental Health problem. The weighted average for Mental Health is 61.53, almost exactly half of the highest score. Twelve of the problems received a weighted average of less than $25 \%$ of the possible points, where another natural gap appears to exist.

\section{Discussion}

Practicing dentists were surveyed regarding problems used in a standardized terminology, The Omaha System, and their possible usefulness in dentistry. All signs/symptoms of the Omaha System were selected by at least one respondent. The results of the survey were, in some ways, surprising. Dentistry has used traditional treatment recommendations (i.e. get your teeth cleaned and examined every six months) for many years. Additionally, there are a finite number of treatments that are frequently provided [3], with few variations in why they were provided or the actual treatment approach itself. The survey, however, suggests that dentists either want to know or believe that knowing more about other issues of patients, represented by problems in the Omaha System, would be useable information. This may suggest that additional information from other domains identified within the Omaha System could influence decisions on treatment frequency and protocol.

Income and nutrition factors were rated above the midpoint by the respondents. Abuse also was above the mid-point and all three of these problems were considered more important than personal care. Given the emphasis of most dental offices concerning home care, this may be an important finding. 
The lack of diagnostic codes greatly limits the ability of researchers to relate disease to treatment and evaluate varying treatment modalities. Additionally, many restorative treatments in dentistry tend to last for years, making studies utilizing outcomes something that may not happen in the near future. The current diagnostic terminology used in the US by third party payers is the International Classification of Disease (ICD-9-CM). It is not particularly granular for dentistry. ICD-10-CM has better granularity for dentistry, but is still limited. With the delay of the use of ICD-10-CM until October of 2014 and a moratorium on changes to it, dentistry cannot expect to have effective diagnostic codes and capture mechanisms.

Consideration of other factors thought to be related to dental conditions becomes more critical, if the goal is to improve oral health while containing costs. Analyzing preventive services and standardized protocols in relation to disease reduction may be more achievable and realistic. A potential advantage of the Omaha System is that it offers an outcomes assessment for dental professionals delivering preventive services with respect to both problems identified and interventions used.

For at least some dental diseases that are considered to be largely preventable, such as dental caries, there appears to be a relatively strong stratification along income lines. Identifying other contributing factors is made more complex by the inability to use current data sources to sort by socioeconomic status as well as the commonly accepted factors of nutrition and personal care. A system that can allow for better identification of all of these factors may allow for research that can be more highly refined, possibly resulting in treatment recommendations that may differ based on a patient's background. Certainly public health measures may be more readily adapted if variations are seen in the research results.

General dentistry's lack of familiarity with terminologies other than procedure codes creates a significant educational hurdle. At the same time, there is a very real opportunity and need to develop a system or systems that can be inclusive of proper EHR documentation, necessary claims/payment reporting, and to allow for efficient research that can provide for effective treatment recommendations and regular protocol. Assuming that costs likely influence a patient's choice of care received, having information available about the different backgrounds of patients could be valuable for both treatment and research purposes.

Conceivably, all 42 problems in the Omaha System Problem Scheme could be included in the information obtained from a patient for use within an electronic records system [10, 11]. Most dental practice management systems can be readily adapted to include the list. However, it may prove to have greater usage and function if the list is limited. Workflow considerations would be critical and a study of how it would function clinically would be an appropriate early design step. The authors believe that practitioners might not complete the entire problem list. Therefore, a prioritized list has been developed from the results of the survey. It can be found in Figure 1 . This would allow offices to more readily select information they consider most relevant, while allowing other offices to use the comprehensive scheme, if desired.

\section{Conclusion}

Regardless of whether the Omaha System is further developed for dentistry, dentistry will benefit from the knowledge acquired in this survey. The determination of problems, signs and symptoms that dentists consider important and would use suggests that these need to be included in any system eventually used by members of the profession for EHR documentation.

Preventive dental services account for a very large proportion of care delivered by dental professionals and costs in the delivery system. Dentistry has limited information on the most effective preventive protocols for disease reduction. A system that can obtain information in a structured form could lead to development of these protocols. Further research, relating the intervention and outcomes components to the problems would be necessary in order to determine whether this tool can assist in defining best preventive practices for populations. 


\section{Clinical Relevance Statement}

Identification of a system that can relate problems to outcomes in a structured way would be new to dentistry. It could allow for the development of "best practices" and decision support tools to assist practitioners in providing the best possible care to patients.

\section{Conflict of Interest}

The authors declare that they have no conflicts of interest in the research.

\section{Human Subjects Protections}

This research used an anonymous survey. No human and/or animal subjects were included in any form of clinical research. An exemption was received from the University of Minnesota Institutional Review Board. 


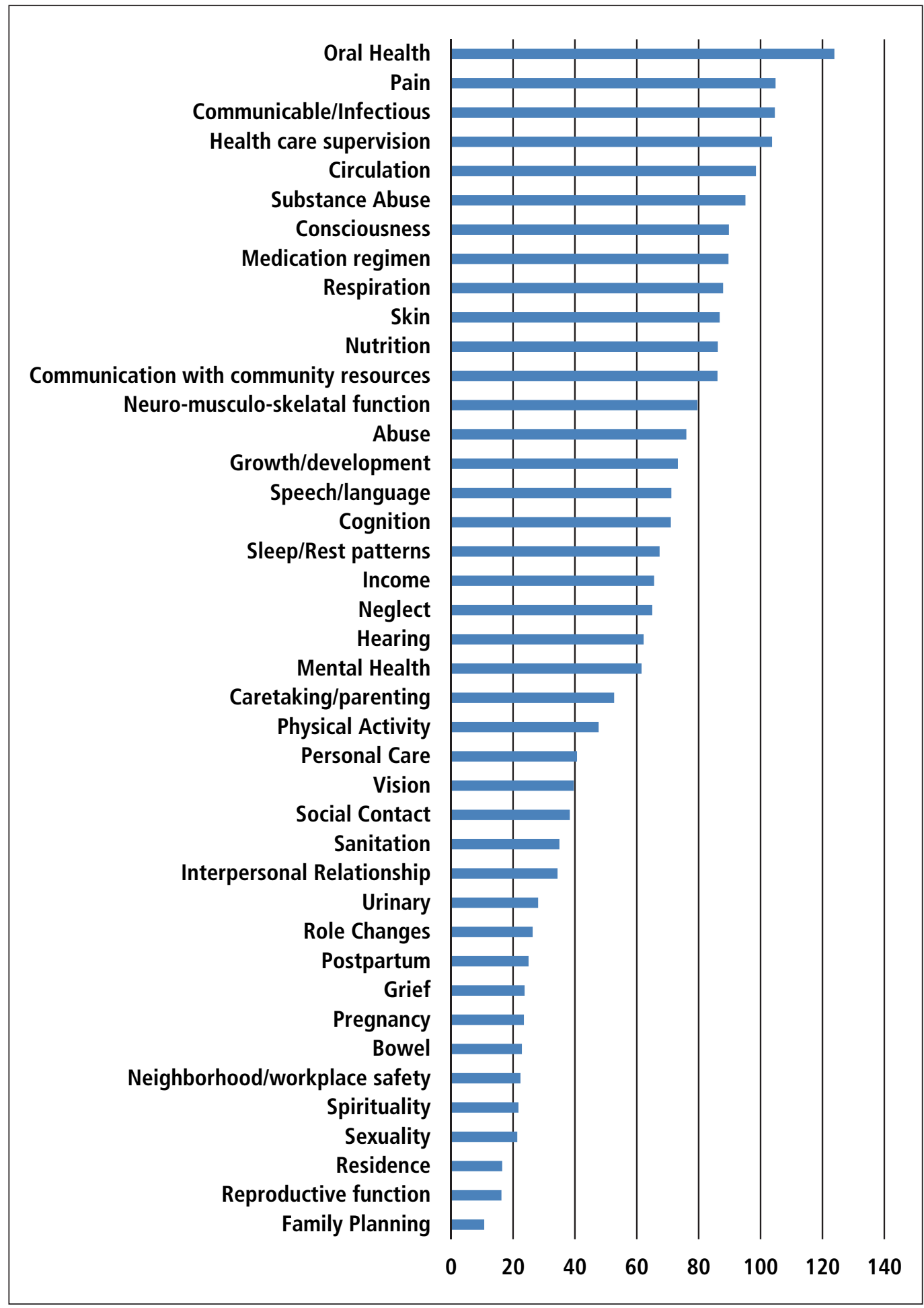

Fig. 1 Problem Rating Summary Totals 
Table 1 Example of the Problem Classification for Oral Health from the Omaha System

\begin{tabular}{|l|l|l|l|}
\hline 1.Missing/broken/malformed teeth & I would use & Others might use & Not useful \\
\hline 2. Caries & & & \\
\hline 3. Excess Tartar & & \\
\hline 4. Sore/swollen/bleeding gums & & \\
\hline 5. Malocclusion & & \\
\hline 6. Ill-fitting/missing dentures & & \\
\hline 7. Sensitivity to hot or cold & & \\
\hline
\end{tabular}

Table 2 Applying the Omaha System Problem Classification to Dental Caries: This is an example of the Omaha System as it applies to dental caries. This example indicates the granularity that can be achieved with the Omaha System, but also indicates that additional refinement for dental specific conditions and care may be beneficial. A dentist diagnoses evident decay on tooth \#3 distal and treatment plans a restoration 3 DO, after patient reports temperature sensitivity in the upper right quadrant.

\begin{tabular}{|l|l|l|l|l}
\hline Domain & Physiological \\
\hline Problem & $\begin{array}{l}\text { Oral Health } \\
\text { Modifiers: Individual and Actual }\end{array}$ \\
\hline Signs/Symptoms of Actual & $\begin{array}{l}\text { Caries (\#3 distal) } \\
\text { Sensitivity to hot/cold }\end{array}$ \\
\hline Intervention Scheme & $\begin{array}{l}\text { Category: Teaching, Guidance, and Counseling } \\
\text { - Dietary Management (patient needs to reduce soda intake from } 4 \\
\text { bottles/day) } \\
\text { - Personal hygiene, frequent brushing and flossing (2x/fay } 3 \text { mins } \\
\text { brush each time, floss before bedtime) } \\
\text { - Supplies toothbrush (extra soft), floss (waxed, ribbon) } \\
\text { Category: Treatment and Procedures } \\
- \text { Medical/dental care } \\
\text { - c. fill caries } \\
\text { Category: Case Management } \\
- \text { Medical/dental care } \\
\text { - b. schedule/provide services (patient scheduled necessary appoint- } \\
\text { ment) } \\
\text { - Surveillance } \\
\text { - Medical/dental care }\end{array}$ \\
\hline Receives care when scheduled & \\
\hline Follows plan of care & \\
\hline
\end{tabular}


Table 3 Weighted results of survey by Omaha System Problem Classification

\begin{tabular}{|c|c|c|c|c|c|}
\hline Problem & Would Use & Others Might & Total & $\begin{array}{l}\text { \#Signs/ } \\
\text { Symptoms }\end{array}$ & Average \\
\hline Family Planning & 0 & 64 & 64 & 6 & 10.67 \\
\hline Reproductive Function & 36 & 78 & 114 & 7 & 16.25 \\
\hline Residence & 57 & 174 & 231 & 14 & 16.50 \\
\hline Sexuality & 81 & 90 & 171 & 8 & 21.38 \\
\hline Spirituality & 39 & 48 & 87 & 4 & 21.75 \\
\hline Neighborhood/Workplace Safety & 111 & 113 & 224 & 10 & 22.40 \\
\hline Bowel & 81 & 79 & 160 & 7 & 22.86 \\
\hline Pregnancy & 72 & 69 & 141 & 6 & 23.50 \\
\hline Grief & 54 & 41 & 95 & 4 & 23.75 \\
\hline Postpartum & 84 & 66 & 150 & 6 & 25.00 \\
\hline Role Changes & 42 & 37 & 79 & 3 & 26.33 \\
\hline Urinary & 153 & 100 & 253 & 9 & 28.11 \\
\hline Interpersonal Relationship & 192 & 83 & 275 & 8 & 34.38 \\
\hline Sanitation & 222 & 163 & 385 & 11 & 35.00 \\
\hline Social Contact & 60 & 55 & 115 & 3 & 38.33 \\
\hline Vision & 204 & 113 & 317 & 8 & 39.63 \\
\hline Personal Care & 258 & 108 & 366 & 9 & 40.67 \\
\hline Physical Activity & 93 & 50 & 143 & 3 & 47.67 \\
\hline Caretaking/parenting & 360 & 114 & 474 & 9 & 52.67 \\
\hline Mental Health & 819 & 227 & 1046 & 17 & 61.53 \\
\hline Hearing & 261 & 50 & 311 & 5 & 62.20 \\
\hline Neglect & 309 & 81 & 390 & 6 & 65.00 \\
\hline Income & 261 & 67 & 328 & 5 & 65.60 \\
\hline Sleep/Rest Patterns & 447 & 92 & 539 & 8 & 67.38 \\
\hline Cognition & 600 & 110 & 710 & 10 & 71.00 \\
\hline Speech/language & 369 & 58 & 427 & 6 & 71.17 \\
\hline Growth/development & 246 & 47 & 293 & 4 & 73.25 \\
\hline Abuse & 522 & 86 & 608 & 8 & 76.00 \\
\hline Neuro-musculo-skeletal function & 945 & 90 & 1035 & 13 & 79.62 \\
\hline $\begin{array}{l}\text { Communication w/community re- } \\
\text { sources }\end{array}$ & 840 & 107 & 947 & 11 & 86.09 \\
\hline Nutrition & 726 & 136 & 862 & 10 & 86.20 \\
\hline Skin & 777 & 91 & 868 & 10 & 86.80 \\
\hline Respiration & 795 & 84 & 879 & 10 & 87.90 \\
\hline Medication regimen & 642 & 75 & 717 & 8 & 89.63 \\
\hline Consciousness & 327 & 32 & 359 & 4 & 89.75 \\
\hline Substance Abuse & 774 & 82 & 856 & 9 & 95.11 \\
\hline
\end{tabular}


Table 3 Continued

\begin{tabular}{|l|c|c|c|c|l|}
\hline Problem & Would Use & Others Might & Total & $\begin{array}{l}\text { \#Signs/ } \\
\text { Symptoms }\end{array}$ & Average \\
\hline Circulation & 1467 & 109 & 1576 & 16 & 98.50 \\
\hline Health Care Supervision & 675 & 51 & 726 & 7 & 103.71 \\
\hline Communicable/Infectious & 786 & 51 & 837 & 8 & 104.62 \\
\hline Pain & 594 & 35 & 629 & 6 & 104.83 \\
\hline Oral Health & 964 & 3 & 967 & 7 & 123.86 \\
\hline
\end{tabular}




\section{References}

1. White JM, et al. Evaluating a dental diagnostic terminology in an electronic health record. J Dent Educ 2011: 75(5): 605-615.

2. Kalenderian E, et al. The development of a dental diagnostic terminology. J Dent Educ 2011: 75(1): 68-76.

3. National Association of Dental Plans. Statistical review of member reports, 2012. Accessed July 2013.

4. Lewis C, Stout J. Toothaches in U.S. Children. Archives of Pediatric Adolescent Medicine 2010; 164: 1059-1063.

5. U.S. General Accounting Office. Extent of dental disease in children has not decreased, and million are estimated to have untreated tooth decay. GAO-08-1121. Washington, DC: U.S > General Accounting Office, September 2008

6. Current Dental Terminology 2011. American Dental Association: 2010.

7. Palmer C. Dental Quality Alliance role needed for ADA implementation. American Dental Association web site. http://www.ada.org/news/7252.aspx. Accessed September 2012.

8. Benson, T. Principles of Health Interoperability, HL7 and SNOMED: 2010.

9. Wall TP, Vujicic M, Nasseh K. Recent trends in utilization of dental care in the United States. J Dent Educ 2012: 76(8): 1020-1027.

10. Martin KS. The Omaha System: A key to practice, documentation and information management. 2nd ed. Omaha, NE: Health Connection Press: 2009: 484.

11.The Omaha System. http://omahasystem.org/ Accessed August 2012.

12. SurveyMonkey. www.surveymonkey.com Accessed August 2012.

13.ICD10CM_2012 - U.S. Department of Health and Human Services, Centers for Medicare \& Medicaid Services; ICD-10_CM [computer file]: International Classification of Disease. Tenth Revision. Clinical Modification: December 1, 2011: Hyattsville, MD; provided for public viewing only, and not valid for use prior to October 1, 2013.

14.ICD9CM 2013(updated) - U.S. Department of Health and Human Services, Centers for Medicare \& Medicaid Services; ICD-10_CM [computer file]: International Classification of Disease. Ninth Revision. Clinical Modification: October 1, 2012: Baltimore, MD. 


\section{Appendix 1}

Dear Doctor (name),

Today, dentistry lacks a terminology that allows us to effectively describe many symptoms, diagnosis and problems we see among our patients. Electronic dental records have the potential to improve outcomes and allow for more efficient and effective communication. However, without appropriate and complete terminology, the potential is limited. If you have ever submitted a claim to a medical insurance plan, it is evident how the terminology they accept is difficult to use for dental issues.

While we currently describe what procedures we perform with CDT codes, much of the remaining information we obtain at every appointment is locked into words that we use in our clinical notes. These words can vary dramatically and are much more difficult to recover from the data than a new terminology might be.

We are asking you to take a few minutes to fill out the survey on the link below. The survey is looking at a terminology that has been used in the medical care area for about 25 years and may provide better descriptions of what a dental clinician may wish to consider prior to beginning treatment.

This survey looks at the problem list of a terminology known as the Omaha System. The Omaha System also includes areas for interventions (treatments, procedures, etc) and one for outcomes evaluation, but those are beyond the scope of this survey. This survey simply would allow us to identify all of the possible problems, signs and symptoms that you might want to document somewhere within a patient record.

The survey, though somewhat lengthy, is quite simple. You are asked to merely check any of the problems, conditions, environment, signs or symptoms that might apply to a patient or have an impact on a treatment or outcome for a patient. If it is something you might put somewhere into a patient's chart, even if it is just a single patient, you should check it.

There is one area in the survey that pertains directly to oral health. Other areas may be very beneficial to be able to document somewhere in our dental records and others likely won't apply. The Omaha System is designed to reach across all health care related services with a common format and provides a way to document many aspects about a patient in a more structured way. Thus, some of the items in the problem list may seem very remote to dentistry, at best.

The results of this survey and possible next steps in terminology development will be openly available to anyone interested in either looking at, or using the data. Also, the information you submit will have no specific identifiers and therefore, submission is anonymous.

Please take the time to help us with this research. The survey needs to close on 7/8/12.

If you have any questions or comments about the survey instrument, please do not hesitate to contact me. Thank you in advance for your time and assistance!

Mark W Jurkovich DDS

jurko003@umn.edu 